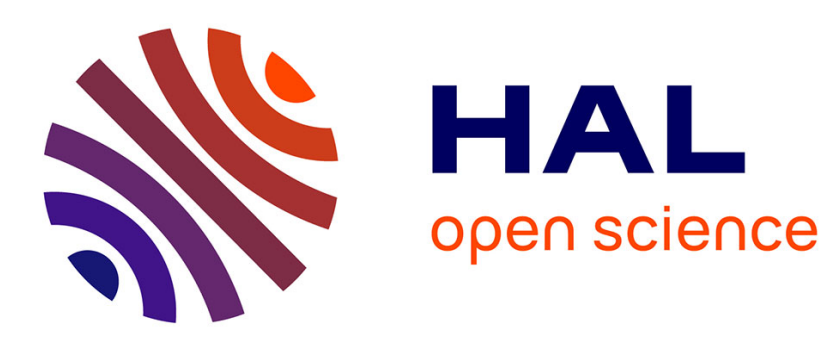

\title{
The cluster model and the generalized Brody-Moshinsky coefficients
}

B. Silvestre-Brac

\section{To cite this version:}

B. Silvestre-Brac. The cluster model and the generalized Brody-Moshinsky coefficients. Journal de Physique, 1985, 46 (7), pp.1087-1099. 10.1051/jphys:019850046070108700 . jpa-00210052

\section{HAL Id: jpa-00210052 https://hal.science/jpa-00210052}

Submitted on 1 Jan 1985

HAL is a multi-disciplinary open access archive for the deposit and dissemination of scientific research documents, whether they are published or not. The documents may come from teaching and research institutions in France or abroad, or from public or private research centers.
L'archive ouverte pluridisciplinaire HAL, est destinée au dépôt et à la diffusion de documents scientifiques de niveau recherche, publiés ou non, émanant des établissements d'enseignement et de recherche français ou étrangers, des laboratoires publics ou privés. 
Classification

Physics Abstracts

$21.60 \mathrm{G}$

\title{
The cluster model and the generalized Brody-Moshinsky coefficients
}

\author{
B. Silvestre-Brac \\ Institut des Sciences Nucléaires, 53 Avenue des Martyrs, 38026 Grenoble Cedex, France
}

(Reçu le 26 novembre 1984, accepté le $1^{\mathrm{er}}$ mars 1985)

\begin{abstract}
Résumé. - Les théories d'agrégats éliminant rigoureusement le mouvement du centre de masse font appel aux coordonnées intrinsèques des agrégats. On montre que les coordonnées de Jacobi des différents agrégats sont reliées par une transformation orthogonale et que l'utilisation des coefficients de Brody-Moshinsky généralisés permet le calcul exact des noyaux d'échange. La procédure est illustrée dans le cas de la description de l'interaction nucléon-nucléon en termes de quarks constituants.
\end{abstract}

Abstract. - Cluster theories, which rigorously eliminate the centre of mass motion, need intrinsic cluster coordinates. It is shown that the Jacobi coordinates of the various clusters are related by an orthogonal transformation and that the use of generalized Brody-Moshinsky coefficients allows an exact calculation of the exchange kernels. This procedure is illustrated by the description of nucleon-nucleon interaction in terms of constituent quarks.

\section{Introduction.}

The non-relativistic problem of many interacting particles - even in the simplest case of two-body forces only - has attracted a large number of studies for the last thirty years and has initiated many sophisticated methods. Apart from the two body problem whose Schrödinger equation reduces to an ordinary differential equation, only the three body problem was treated with sufficient accuracy to pretend to be " exactly solved ». The study of four body systems is rapidly progressing and no doubt that their « exact solution " will be a reality very soon. More complicated systems are tackled only through some kinds of approximation. The shell models in terms of independent particle wave functions are widely used because the Pauli principle can be taken into account rather easily (especially if the formalism of second quantization is employed). However, these models violate an essential symmetry of the Hamiltonian : the space translation invariance. One thinks that this violation is not very important for the bound states of heavy systems. On the other hand, a good treatment of the centre of mass motion is crucial for the scattering problem or for the description of light systems. If the independent particle scheme is conserved (in this case, the Pauli principle is easy), the restoration of the broken symmetry is a very complicated task [1]. Another possibility is to use harmonic oscillator functions as a basis of independent particle motion.
In that case the centre of mass motion comes also into a harmonic oscillator motion and to eliminate the spurious centre of mass motion it is necessary to diagonalize matrices bigger than the physical problem due to the possibility of excitation of the centre of mass in several quanta. In fact, the only way to eliminate rigorously the centre of mass motion is to separate the total wave function of the $n$ particles in terms of a centre of mass function $f_{\text {C.M. }}$ (R) (a plane wave for instance) and an intrinsic function $\psi_{\text {int }}\left(\boldsymbol{\rho}_{1}, \ldots, \boldsymbol{\rho}_{n-1}\right)$ expressed through $n-1$ intrinsic coordinates $\rho_{i}$. In that case the antisymmetrization of $\psi_{\text {int }}$ is particularly difficult. As a matter of fact a permutation $P$ of the particles concerns the original coordinates $\mathbf{r}_{i}$ and not the intrinsic coordinates $\boldsymbol{\rho}_{i}$. Consequently, given a trial intrinsic wave function $\psi_{\text {int }}$ not yet antisymmetrized, the Pauli principle requires the evaluation of the so-called exchange kernels

$$
\begin{aligned}
\left\langle P^{\prime} \psi_{\text {int }}\left(\boldsymbol{\rho}_{1}, \ldots, \boldsymbol{\rho}_{n-1}\right)\right| V_{i j}\left(\mathbf{r}_{i}-\right. & \left.\mathbf{r}_{j}\right) \mid \times \\
& \left.\times P \psi_{\text {int }}\left(\boldsymbol{\rho}_{1}, \ldots, \boldsymbol{\rho}_{n-1}\right)\right\rangle
\end{aligned}
$$

this is a terrible task. Usually, one simplifies a bit the problem by choosing trial wave functions which are built on definite eigenmodes of subsystems : this is the essence of the cluster models [2]. This allows a drastic reduction of the Hilbert space while keeping most of the physical correlations; nevertheless the Pauli principle must be fulfilled and the problem of exchange 
kernels still remains. Even in the cluster models, the sub-wave functions are themselves chosen very simple (usually Gaussians) and this may lead to inconsistencies between the dynamical description of the clusters and the total system [13].

In this paper, we present a general method allowing an exact calculation of the exchange kernels, based on the expansion of the intrinsic wave function in terms of independent harmonic oscillator wave functions. The generalized Brody-Moshinsky coefficients are crucial ingredients in this method. In the next section, the relations between cluster internal coordinates and those obtained by permutation of the particles are discussed in detail. In section 3, a number of properties concerning the generalized Brody-Moshinsky coefficients is presented. In section 4 the formalism is applied to the description of the nucleon-nucleon scattering in terms of quark structure. Conclusions are drawn in the last section.

\section{Relations between intrinsic cluster coordinates}

2.1 Definitions. - Let us describe a system of $n$ identical particles. In this paper, only the coordinate degrees of freedom are considered; in fact the spin and isospin degrees of freedom are treated in a straightforward, although lenghtly, way with help of Racah algebra. The particles are thus referred in the ordinary space by their coordinates $\mathbf{r}_{i}(i=1,2, \ldots, n)$ relative to some arbitrary origin. Since the total Hamiltonian is invariant under space translation it is necessary to introduce the centre of mass coordinate

$$
\mathbf{R}=\frac{1}{n} \sum_{i=1}^{n} \mathbf{r}_{i}
$$

Besides, there remains $n-1$ linearly independent coordinates (called intrinsic coordinates); they can be chosen in an infinite number of ways but the most common choice defines the Jacobi coordinates, which represent the distance of the $(i+1)$ th particle relative to the centre of mass of the $i$ previous particles

$$
\boldsymbol{\rho}_{i}=\alpha[i(i+1)]^{-1 / 2}\left(\mathbf{r}_{1}+\mathbf{r}_{2}+\cdots+\mathbf{r}_{i}-i \mathbf{r}_{i+1}\right)
$$

$\alpha$ is a scale parameter whose role will be emphasized later. One can write more formally

$$
\boldsymbol{\rho}_{i}=\alpha \sum_{j=1}^{n} A_{i j} \mathbf{r}_{j} \text { or } \quad(\boldsymbol{\rho})=\alpha A(\mathbf{r})
$$

where $A$ is a $n-1$ by $n$ matrix

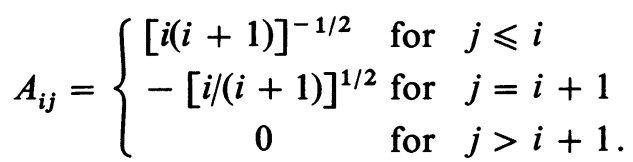

It has the properties

$$
A A^{t}=1_{(n-1) \times(n-1)} \text { and } \sum_{j=1}^{n} A_{i j}=0 \quad \forall i
$$

(note that we do not have $A^{t} A=\mathbb{1}_{n \times n}$ ).
It is simple to invert (2.1) and (2.3) to find

or

$$
\mathbf{r}_{j}=\mathbf{R}+\alpha^{-1} \sum_{i=1}^{n-1} A_{i j} \boldsymbol{\rho}_{i}
$$

$$
\text { (r) }=\mathbf{R}(\mathfrak{\imath})+\alpha^{-1} A^{t}(\mathbf{p})
$$

To achieve a quantum mechanical description of the system we need the canonical momenta of $\mathbf{R}$ and $\rho_{i}$ which are referred to as $\mathbf{P}$ and $\pi_{i}$. Starting from the canonical conjugates $\mathbf{p}_{i}$ of the initial coordinates $\mathbf{r}_{i}$, it is not difficult to show that

$$
\mathbf{P}=\sum_{i=1}^{n} \mathbf{p}_{i} \quad(\boldsymbol{\pi})=\alpha^{-1} A(\mathbf{p})
$$

and the inverse relations

$$
\text { (p) }=n^{-1} \mathbf{P}(\mathbb{1})+\alpha A^{t}(\pi) \text {. }
$$

The kinetic energy $T=\frac{1}{2 m} \sum_{i=1}^{n} \mathbf{p}_{i}^{2}$ can be recast as

$$
T=\frac{\mathbf{P}^{2}}{2 n m}+\frac{\alpha^{2}}{2 m} \sum_{i=1}^{n-1} \pi_{i}^{2} \text {. }
$$

Exactly in the same way we have

$$
\sum_{i=1}^{n} \mathbf{r}_{i}^{2}=n \mathbf{R}^{2}+\alpha^{-2} \sum_{i=1}^{n-1} \rho_{i}^{2}
$$

For a Hamiltonian invariant under space translation, the potential energy does not depend on the $\mathbf{R}$ coordinate, and with the Jacobi coordinates we are left with an interacting system of $n-1$ particles with mass $\frac{m}{\alpha^{2}}$. Thus, $\alpha$ is a scale mass parameter, which is not relevant for the essential features of the problem. The total orbital wave function is separated into a centre of mass part and an intrinsic one.

$\Psi_{\mathbf{K}}\left(\mathbf{r}_{1}, \mathbf{r}_{2}, \ldots, \mathbf{r}_{n}\right)=\exp (-i \mathbf{K} \cdot \mathbf{R}) \psi_{\text {int }}\left(\boldsymbol{\rho}_{1}, \boldsymbol{\rho}_{2}, \ldots, \boldsymbol{\rho}_{n-1}\right)$

2.2 Action of A Permutation. - Since the Pauli principle imposes a complete antisymmetric wavefunction, one needs to know the effect of a permutation $P$ on the orbital wave function. If $P$ is the permutation which changes the $i$ th particle into the $P_{i}$ th particle, the action of $P$ is particularly simple expressed in the original coordinates since $\mathbf{r}_{i} \rightarrow \mathbf{r}_{i}^{(\mathbf{P})}=\mathbf{r}_{P_{i}}$. Thus

$$
\begin{aligned}
P \Psi_{\mathbf{K}}\left(\mathbf{r}_{1}, \mathbf{r}_{2}, \ldots, \mathbf{r}_{n}\right)=\Psi_{\mathbf{K}}\left(\mathbf{r}_{1}^{(\mathrm{P})}, \mathbf{r}_{2}^{(\mathrm{P})}, \ldots, \mathbf{r}_{n}^{(\mathrm{P})}\right)= \\
=\Psi_{\mathbf{K}}\left(\mathbf{r}_{P_{1}}, \mathbf{r}_{P_{2}}, \ldots, \mathbf{r}_{P_{n}}\right)
\end{aligned}
$$

The centre of mass coordinate is symmetric for every permutation so $\mathbf{R}^{(\mathbf{P})}=\mathbf{R}$. On the other hand, the effect of the permutation on the intrinsic wave function is not obvious

$$
P \psi_{\text {int }}\left(\boldsymbol{\rho}_{1}, \boldsymbol{\rho}_{2}, \ldots, \boldsymbol{\rho}_{n-1}\right)=\psi_{\text {int }}\left(\boldsymbol{\rho}_{1}^{(\mathbf{P})}, \boldsymbol{\rho}_{2}^{(\mathbf{P})}, \ldots, \boldsymbol{\rho}_{n-1}^{(\mathbf{P})}\right)
$$

where

$$
\boldsymbol{\rho}_{i}^{(\mathrm{P})}=\alpha \sum_{j=1}^{n} A_{i j} \mathbf{r}_{P_{j}}=\alpha \sum_{j=1}^{n} A_{i P_{\bar{j}}^{-1}} \mathbf{r}_{j}
$$


which is different from :

$$
\boldsymbol{\rho}_{P_{i}}=\alpha \sum_{j=1}^{n} A_{P_{i} P_{j}} \mathbf{r}_{P_{j}}=\alpha \sum_{j=1}^{n} A_{P_{i j}} \mathbf{r}_{j}
$$

Hence, as seen from (2.13), (2.14) and (2.15), the effect of $P$ on the intrinsic wave function is much more complicated than a simple permutation of the intrinsic Jacobi coordinates.

However, it is trivial to show that $\boldsymbol{\rho}^{(\mathbf{P})}$ are also intrinsic coordinates in the sense that they do not depend on the $\mathbf{R}$ coordinate. Hence

$$
\boldsymbol{\rho}_{i}^{(\mathbf{P})}=\sum_{j=1}^{n-1} S_{i j}^{(\mathbf{P})} \boldsymbol{\rho}_{j} \quad \text { or } \quad\left(\boldsymbol{\rho}^{(\mathbf{P})}\right)=S^{(\mathbf{P})}(\boldsymbol{\rho})
$$

From the equality $\sum_{i=1}^{n} \mathbf{r}_{i}^{2}=\sum_{i=1}^{n} \mathbf{r}_{i}^{(\mathbf{P})^{2}}$ and from relation (2.10) one has

$$
\sum_{i=1}^{n-1} \boldsymbol{\rho}_{i}^{\left(\mathbf{P}^{2}\right.}=\sum_{i=1}^{n-1} \boldsymbol{\rho}_{i}^{2}
$$

and hence the permutation matrix $S_{(n-1) \times(n-1)}^{(P)}$ is orthogonal

$$
S^{(\mathbf{P})^{t}} S^{(\mathbf{P})}=S^{(\mathbf{P})} S^{(\mathbf{P})^{t}}=\mathbb{1}_{(n-1) \times(n-1)} .
$$

2.3 Clustering OF THE SYSTEM. - Very often it is advantageous to express the eigenmodes of a system in terms of eigenmodes of sub-systems; in many cases the choice of sub-systems (or clusters) lies on a definite physical situation. Moreover, the restriction to few eigenmodes of the clusters allows a drastic truncation in the total Hilbert space, while retaining most of the relevant correlations. Hence, it is necessary to have the relation between the original Jacobi coordinates for the total system as defined in the previous subsection and the intrinsic coordinates of the clusters. At the moment let us suppose that the $n$-particle system is partitioned in two subsystems of $s_{1}$ and $s_{2}$ particles $\left(s_{i}>1\right.$ and $\left.s_{1}+s_{2}=n\right)$. It is natural to introduce the intrinsic coordinates of the first cluster

$$
\boldsymbol{\rho}_{i}^{[1]}=\alpha[i(i+1)]^{-1 / 2}\left(\mathbf{r}_{1}+\mathbf{r}_{2}+\cdots+\mathbf{r}_{i}-i \mathbf{r}_{i+1}\right) \quad i=1, \ldots, s_{1}-1
$$

and of the second cluster

$$
\boldsymbol{\rho}_{i}^{[2]}=\alpha[i(i+1)]^{-1 / 2}\left(\mathbf{r}_{s_{1}+1}+\mathbf{r}_{s_{1}+2}+\cdots+\mathbf{r}_{s_{1}+i}-i \mathbf{r}_{s_{1}+i+1}\right) \quad i=1, \ldots, s_{2}-1 .
$$

Since only $n-2$ intrinsic coordinates have been defined, we need one more which is taken as the separation distance of the two clusters, namely

$$
\boldsymbol{\rho}_{s_{2}}^{[2]}=\mathbf{d}^{[1,2]}=\alpha\left[n s_{1} s_{2}\right]^{-1 / 2}\left[s_{1}\left(\mathbf{r}_{s_{1}+1}+\cdots+\mathbf{r}_{n}\right)-s_{2}\left(\mathbf{r}_{1}+\cdots+\mathbf{r}_{s_{1}}\right)\right] .
$$

By replacing the $\mathbf{r}_{i}$ in terms of $\boldsymbol{\rho}_{i}$ in these expressions it is just a matter of calculation to find the new Jacobi coordinates as function of the old ones : it comes

$$
\begin{gathered}
\boldsymbol{\rho}_{i}^{[1]}=\boldsymbol{\rho}_{i} \\
\boldsymbol{\rho}_{i}^{[2]}=-s_{1}[i(i+1)]^{-1 / 2} \sum_{j=1}^{i}\left[\left(s_{1}-1+j\right)\left(s_{1}+j\right)\right]^{-1 / 2} \boldsymbol{\rho}_{s_{1}-1+j}+\left[\frac{i\left(s_{1}+i+1\right)}{(i+1)\left(s_{1}+i\right)}\right]^{1 / 2} \boldsymbol{\rho}_{s_{1}+i} \\
\boldsymbol{\rho}_{s_{2}}^{[2]}=\mathbf{d}^{[1,2]}=-\left[n s_{1} s_{2}^{-1}\right]^{1 / 2} \sum_{j=1}^{s_{2}}\left[\left(s_{1}-1+j\right)\left(s_{1}+j\right)\right]^{-1 / 2} \boldsymbol{\rho}_{s_{1}-1+j} .
\end{gathered}
$$

We feel that it is more convenient to write this change of basis in a matricial form; then the cluster coordinates are put in the form

$$
\left(\rho^{[c]}\right) \equiv\left(\begin{array}{c}
\rho^{[1]} \\
\rho^{[2]} \\
d
\end{array}\right)
$$

which is a vector with $n-1$ components and equations (2.22) can be recast as

$$
\left(\rho^{[c]}\right)=F^{[c]}(\rho) \text { with } F^{[c]}=\mathbb{1}_{\left(s_{1}-1\right) \times\left(s_{1}-1\right)}+F_{s_{2} \times s_{2}}^{[2]}
$$

where $F^{[c]}$ is considered as the direct sum of the unity operator in the space spanned by the first $s_{1}-1 \mathrm{Jacobi}$ coordinates and the matrix $F_{s_{2} \times s_{2}}^{[2]}$ in the complementary space. The analytical form for $F^{[2]}$ follows from (2.22)

$$
\begin{aligned}
& F_{i j}^{[2]}=\left\{\begin{array}{cl}
-s_{1}\left[i(i+1)\left(s_{1}-1+j\right)\left(s_{1}+j\right)\right]^{-1 / 2} & \text { for } j<i \\
{\left[i\left(s_{1}+i+1\right) /(i+1)\left(s_{1}+i\right)\right]^{1 / 2}} & \text { for } j=i+1 \\
0 & \text { for } j>i+1
\end{array} \quad i=1,2, \ldots, s_{2}-1\right. \\
& F_{s_{2} j}^{[2]}=-\left[n s_{1} s_{2}^{-1}\right]^{1 / 2}\left[\left(s_{1}+j-1\right)\left(s_{1}+j\right)\right]^{-1 / 2} .
\end{aligned}
$$


From the matrix elements defined by (2.24) one can show that $F^{[2]}$ is an orthogonal matrix, and consequently this property holds too for the matrix $F^{[c]}$ defined by $(2.23)$

$$
F^{[c]^{t}} F^{[c]}=F^{[c]} F^{[c]^{t}}=\mathfrak{1}_{(n-1) \times(n-1)} .
$$

The generalization to an arbitrary number of clusters is obtained by recurrence. Let us suppose that $s_{1}$ is now partitioned into two clusters, then the new first cluster is divided into two further clusters, and so on. Thus one considers the partition of the original system in $k$ clusters, each one having $s_{i}$ particles $\left(s_{i}>1, s_{1}+s_{2}+\cdots+s_{k}=n\right)$. The various partitions of the total system play a very important role in any manybody theories eliminating correctly the centre of mass motion [3] (see for instance the Yakubovski equations for the four-body problem [4]).

To simplify the notation it is convenient to define

$$
t_{l}=s_{1}+s_{2}+\cdots+s_{l-1} \text { and by convention } t_{k+1}=n, t_{1}=0 .
$$

The intrinsic Jacobi coordinates for the $l$ th cluster are

$$
\begin{aligned}
& \boldsymbol{\rho}_{i}^{[l]}=\alpha[i(i+1)]^{-1 / 2}\left(\mathbf{r}_{t_{l}+1}+\mathbf{r}_{t_{l}+2}+\cdots+\mathbf{r}_{t_{l}+i}-i \mathbf{r}_{t_{l}+i+1}\right) \\
& \pi_{i}^{[l]}=\alpha^{-1}[i(i+1)]^{-1 / 2}\left(\mathbf{p}_{t_{l}+1}+\mathbf{p}_{t_{l}+2}+\cdots+\mathbf{p}_{t_{l}+i}-i \mathbf{p}_{t_{l}+i-1}\right)
\end{aligned}
$$

and the separation coordinates

$$
\begin{aligned}
& \rho_{s_{l}}^{[l]}=\mathbf{d}^{\left[l, t_{l}\right]}=\alpha\left[t_{l+1} t_{l} s_{l}\right]^{-1 / 2}\left[t_{l}\left(\mathbf{r}_{t_{l}+1}+\cdots+\mathbf{r}_{t_{l+1}}\right)-s_{l}\left(\mathbf{r}_{1}+\cdots+\mathbf{r}_{t_{t}}\right)\right] \\
& \pi_{s_{l}}^{[l]}=\mathbf{q}^{\left[l, t_{l}\right]}=\alpha^{-1}\left[\begin{array}{ll}
t_{l+1} & t_{l} s_{l}
\end{array}\right]^{-1 / 2}\left[t_{l}\left(\mathbf{p}_{t_{l}+1}+\cdots+\mathbf{p}_{t_{l+1}}\right)-s_{l}\left(\mathbf{p}_{1}+\cdots+\mathbf{p}_{t_{l}}\right)\right]
\end{aligned}
$$

then these new intrinsic coordinates are related to the old ones through

$$
\rho_{i}^{[l]}=\sum_{j=1}^{s_{l}} F_{i j}^{[l]} \rho_{t_{l}-1+j}
$$

with the matrix $F_{s_{l} \times s_{l}}^{[l]}$ defined by

$$
\begin{aligned}
& F_{i j}^{[l]}\left\{\begin{array}{cc}
-t_{l}\left[i(i+1)\left(t_{l}-1+j\right)\left(t_{l}+j\right)\right]^{-1 / 2} & \text { for } j \leqslant i \\
{\left[i\left(t_{l}+i+1\right) /(i+1)\left(t_{l}+i\right)\right]^{1 / 2}} & \text { for } j=i+1 \\
0 & \text { for } j>i+1
\end{array}\right. \\
& F_{s_{l} j}^{[l]}=-\left[t_{l+1} t_{l} s_{l}^{-1}\right]^{1 / 2}\left[\left(t_{l}-1+j\right)\left(t_{l}+j\right)\right]^{-1 / 2}
\end{aligned}
$$

As usual, one can write in a more formal way

$$
\left(\rho^{[c]}\right)=\left(\begin{array}{l}
\rho^{[1]} \\
\vdots \\
\rho^{[l]} \\
\vdots \\
\rho^{[k]}
\end{array}\right) ; \quad F_{(n-1) \times(n-1)}^{[c]}=\mathcal{N}_{\left(s_{1}-1\right) \times\left(s_{1}-1\right)}+F_{s_{2} \times s_{2}}^{[2]}+\cdots+F_{s_{l} \times s_{l}}^{[l]}+\cdots+F_{s_{k} \times s_{k}}^{[k]}
$$

then

$$
\begin{aligned}
& \left(\rho^{[c]}\right)=F^{[c]}(\rho) \quad \text { and in the very same way } \\
& \left(\pi^{[c]}\right)=F^{[c]}(\pi) .
\end{aligned}
$$

Since each sub-matrix $F^{[l]}$ is orthogonal, this is also true for the total transformation matrix $F^{[c]}$

$$
F^{[c]^{t}} F^{[c]}=F^{[c]} F^{[c]^{t}}=\mathbb{1}_{(n-1) \times(n-1)} .
$$

Now, we are able to demonstrate the general theorem concerning the intrinsic Jacobi coordinates.

Theorem : Any arbitrary permuted intrinsic coordinate of an arbitrary cluster system is related to another one by an orthogonal transformation (not necessarily a proper orthogonal). 
Proof : Starting from the original coordinates $(\rho)$, one builts the first permuted coordinates $\left(\rho^{(P)}\right)=S^{(\mathbf{P})}(\rho)$ and then the cluster permuted coordinates

Consequently

$$
\left(\rho^{(\mathbf{P})[c]}\right)=F^{[c]}\left(\rho^{(\mathbf{P})}\right)=F^{[c]} S^{(\mathbf{P})}(\boldsymbol{\rho}) \quad \text { or } \quad(\boldsymbol{\rho})=S^{(\mathbf{P})^{t}} F^{[c]^{t}}\left(\boldsymbol{\rho}^{(\mathbf{P})[c]}\right) .
$$

$$
\left(\boldsymbol{\rho}^{(\mathbf{P})[c]}\right)=F^{[c]} S^{(\mathbf{P})} S^{\left(\mathbf{P}^{\prime}\right) t} F^{\left[c^{\prime}\right]^{t}}\left(\boldsymbol{\rho}^{\left(\mathbf{P}^{\prime}\right)\left[c^{\prime}\right]}\right) .
$$

Since each matrix appearing in (2.32) is an orthogonal matrix, their product is also an orthogonal matrix Q.E.D.

As is well known, any orthogonal matrix in $n$ dimensions can be expressed as the product of orthogonal matrices which leaves an $n-2$ dimensional subspace invariant (in other words, any " rotation " in an $n$ dimensional space is obtained by successive "rotations" in fixed planes). Each infinitesimal generator of $0(n)$ is a rotation in two dimensions, and $\frac{n(n-1)}{2}$ generators are enough to generate any general rotation. Thus $\frac{n(n-1)}{2}$ is the maximum number of "plane rotations" necessary to built the original orthogonal matrix. However, it is interesting to search for the " best way " to do the decomposition - that is the one which minimizes the number of operations. The procedure to do that is not unique, and each particular case requires a special treatment to find " the best way ". The cluster wave function being decomposed into independent particle wavefunctions, the problem of evaluating the exchange kernels reduces to expressing two independent functions of rotated coordinates in terms of the functions in the original coordinates. This last problem can be solved if the cluster function is expanded into a harmonic oscillator basis.

\section{Generalized Brody-Moshinsky coefficients.}

3.1 Definitions. - The harmonic oscillator (HO) wave functions $\phi_{n l m}(\mathbf{r})$ are defined with the normalization and phase conventions of Moshinsky [5] or Erdelyi et al. [6]

$$
\phi_{n l m}(\mathbf{r})=b^{-3 / 2} R_{n l}\left(\frac{r}{b}\right) Y_{l m}(\hat{\mathbf{r}})
$$

where $b$ is the size parameter $b=(\hbar / M \omega)^{1 / 2}$, and the radial part is written

$$
R_{n l}(x)=\frac{U_{n l}(x)}{x}=\left[\frac{2(n !)}{\Gamma(n+l+3 / 2)}\right]^{1 / 2} x^{l} e^{-\frac{x^{2}}{2}} L_{n}^{l+1 / 2}\left(x^{2}\right),
$$

and $L_{n}^{l+1 / 2}(x)$ is a generalized Laguerre polynomial. Let us consider the Hamiltonian of two independent harmonic oscillators

$$
H=\frac{1}{2 M}\left(\mathbf{p}_{1}^{2}+\mathbf{p}_{2}^{2}\right)+\frac{1}{2} M \omega^{2}\left(\mathbf{r}_{1}^{2}+\mathbf{r}_{2}^{2}\right) .
$$

An eigenfunction with angular momentum $\lambda$ is readily $\left[\phi_{n_{1} l_{1}}\left(\mathbf{r}_{1}\right) \phi_{n_{2} l_{2}}\left(\mathbf{r}_{2}\right)\right]_{\lambda \mu}$ with energy

$$
\hbar \omega\left(2 n_{1}+l_{1}+2 n_{2}+l_{2}\right) \text {. }
$$

The Hamiltonian (3.3) is invariant under the rotation of the coordinates

$$
\left\{\begin{array} { l } 
{ \mathbf { r } = C \mathbf { r } _ { 1 } - S \mathbf { r } _ { 2 } } \\
{ \mathbf { R } = S \mathbf { r } _ { 1 } + C \mathbf { r } _ { 2 } }
\end{array} \text { or } \quad \left\{\begin{array}{l}
\mathbf{r}_{1}=C \mathbf{r}+S \mathbf{R} \\
\mathbf{r}_{2}=-S \mathbf{r}+C \mathbf{R}
\end{array}\right.\right.
$$

with $C=\cos \frac{\beta}{2}$ and $S=\sin \frac{\beta}{2}$.

Consequently $\left[\phi_{n l}(\mathbf{r}) \phi_{N L}(\mathbf{R})\right]_{\lambda \mu}$ is also an eigenvector with energy $\hbar \omega(2 n+l+2 N+L)$.

Hence one can write

$$
\begin{aligned}
& {\left[\phi_{n_{1} l_{1}}\left(\mathbf{r}_{1}\right) \phi_{n_{2} l_{2}}\left(\mathbf{r}_{2}\right)\right]_{\lambda \mu}=} \\
& =\sum_{n, l, N, L}\left\langle n l N L ; \lambda \mid n_{1} l_{1} n_{2} l_{2} ; \lambda\right\rangle_{\beta}\left[\phi_{n l}(\mathbf{r}) \phi_{N L}(\mathbf{R})\right]_{\lambda \mu} .
\end{aligned}
$$

It is important to note that the summation contains a finite number of terms, namely all the $(n, l, N, L)$ multiplets compatible with the conservation of energy $2 n_{1}+l_{1}+2 n_{2}+l_{2}=2 n+l+2 N+L$ and constraint of the resultant angular momentum $|l-L| \leqslant \lambda \leqslant l+L$ and parity $(-1)^{l_{1}+l_{2}}=(-1)^{l+L}$. The transformation coefficients $\langle n l N L ; \lambda| n_{1} l_{1} n_{2} l_{2}$, $\lambda\rangle_{\beta}$ are called Brody-Moshinsky (BM) coefficients with angle $\beta$. The standard $B M$ coefficients, corresponding to

$$
\beta=\frac{\pi}{2}\left(\text { i.e. } \mathbf{r}=\frac{1}{\sqrt{2}}\left(\mathbf{r}_{1}-\mathbf{r}_{2}\right) \text { and } \mathbf{R}=\frac{1}{\sqrt{2}}\left(\mathbf{r}_{1}+\mathbf{r}_{2}\right)\right),
$$

play a very important role in numerous fields in physics, especially in the expression of matrix elements of two-body effective interactions [7]. Their importance was first recognized by Talmi [15] who calculated some of them by hand. Later on, Moshinsky [5] studied their properties in more detail and in collaboration with Brody [7] made extensive tables. Then, a number of works has been devoted to find new relations between them and to refine and simplify their calculation (see Appendix 6 of Lawson's book [7]). Curiously, since their introduction by Moshinsky [8], the $B M$ coefficients with an angle $\beta \neq \pi / 2$, have suffered from a complete lack of interest, and, as far 
as we are aware of, were very scarcely used in practical cases. In this section, we give the main properties of these $B M$ coefficients and show how useful they are in the cluster theory.

3.2 Calculations. - The standard $B M$ were calculated in several different ways : recurrence relations $[5,9]$, more or less sophisticated analytical formulae. [10], diagonalization of special matrices [11]. In fact, all these methods can be as well applied to the $B M$ with angle $\beta$. This property was not realized first since, in his original paper [8], Moshinsky proposed a complicated formula including the standard coefficients; readily it is not a very convenient one. Which method to choose for computing them depends on what one wants. If one is interested in particular, unrelated coefficients, it is preferable to use analytical formulae; if one wants to evaluate entire lines of the
$B M$ matrices the method proposed by Raynal [12] is certainly better; if on the other hand, one is interested in the whole matrix, or all the $B M$ up to a given number of quanta the diagonalization or the recurrence methods are preferable. The latter was used for the application to the nucleon-nucleon interaction presented in the next section. Thus, here, the $B M$ coefficients are calculated by recurrence relations in the same way as the original standard coefficients. In that sense, all the $B M$ coefficients are treated on an equal footing. Since the method is not new and follows exactly the same steps as in the original work of Moshinsky [5], we do not insist on it. However, the final results never appeared in the literature, as far as we know, and we think that it is interesting to report them. In a first step, the particular $\langle n l N L$; $\lambda\left|n_{1} l_{1} n_{2} l_{2} ; \lambda\right\rangle_{\beta}$ coefficients with $n_{1}=n_{2}=0$ are calculated

$$
\begin{array}{r}
\left\langle n l N L ; \lambda \mid 0 l_{1} 0 l_{2} ; \lambda\right\rangle_{\beta}= \\
=(-1)^{n+l+L+\lambda} 2^{n+N}\left[(2 l+1)(2 L+1) \frac{l_{1} ! l_{2} !}{\left(2 l_{1}\right) !\left(2 l_{2}\right) !} \frac{(n+l) !}{n !(2 n+2 l+1) !} \frac{(N+L) !}{N !(2 N+2 L+1) !}\right]^{1 / 2} \\
\times \sum_{x}(2 x+1)\left\{\begin{array}{lll}
l & L & \lambda \\
l_{2} & l_{1} & x
\end{array}\right\} A\left(l_{1} l, l_{2} L, x ; \beta\right)
\end{array}
$$

with

$$
\begin{gathered}
A\left(l_{1} l, l_{2} L, x ; \beta\right)=\left[\frac{\left(l_{1}+l+x+1\right) !\left(l_{1}+l-x\right) !\left(l_{1}+x-l\right) !}{\left(l+x-l_{1}\right) !}\right]^{1 / 2}\left[\frac{\left(l_{2}+L+x+1\right) !\left(l_{2}+L-x\right) !\left(l_{2}+x-L\right) !}{\left(L+x-l_{2}\right) !}\right]^{1 / 2} \times \\
\quad \times \sum_{q}(-1)^{\frac{1}{2}\left(l+q-l_{1}\right)} C^{l_{1}+l_{2}-q} S^{q} \frac{\left(l+q-l_{1}\right) !}{\left(\frac{l+q-l_{1}}{2}\right) !\left(\frac{l+l_{1}-q}{2}\right) !} \frac{\left(L+q-l_{2}\right) !}{\left(\frac{L+q-l_{2}}{2}\right) !\left(\frac{L+l_{2}-q}{2}\right) !} \frac{1}{(q-x) !(q+x+1) !} .
\end{gathered}
$$

Then, other coefficients with $n_{1} \neq 0$ are obtained by recurrence relations

$$
\begin{aligned}
& {\left[\left(n_{1}+1\right)\left(n_{1}+l_{1}+3 / 2\right)\right]^{1 / 2}\left\langle n l N L ; \lambda \mid n_{1}+1 l_{1} n_{2} l_{2} ; \lambda\right\rangle_{\beta}=C^{2}[n(n+l+1 / 2)]^{1 / 2}\left\langle n-1 l N L ; \lambda \mid n_{1} l_{1} n_{2} l_{2} ; \lambda\right\rangle_{\beta}} \\
& +S^{2}[N(N+L+1 / 2)]^{1 / 2}\left\langle n l N-1 L ; \lambda \mid n_{1} l_{1} n_{2} l_{2} ; \lambda\right\rangle_{\beta} \\
& +C . S\left[\frac{n N(l+L+\lambda+2)(l+L+\lambda+3)(l+L-\lambda+1)(l+L-\lambda+2)]^{1 / 2}\left\langle n-1 l+1 N-1 L+1 ; \lambda \mid n_{1} l_{1} n_{2} l_{2} ; \lambda\right\rangle_{\beta}}{(2 l+1)(2 l+3)(2 L+1)(2 L+3)}\right]^{1 / 2} \\
& +C . S\left[\frac{n(N+L+1 / 2)(L+\lambda-l-1)(L+\lambda-l)(l+\lambda-L+1)(l+\lambda-L+2)}{(2 l+1)(2 l+3)(2 L-1)(2 L+1)}\right]^{1 / 2}\left\langle n-1 l+1 N L-1 ; \lambda \mid n_{1} l_{1} n_{2} l_{2} ; \lambda\right\rangle_{\beta} \\
& +C . S\left[\frac{N(n+l+1 / 2)(l+\lambda-L-1)(l+\lambda-L)(L+\lambda-l+1)(L+\lambda-l+2)}{(2 L+1)(2 L+3)(2 l-1)(2 l+1)}\right]^{1 / 2}\left\langle n l-1 N-1 L+1 ; \lambda \mid n_{1} l_{1} n_{2} l_{2} ; \lambda\right\rangle_{\beta} \\
& +C . S\left[\frac{(n+l+1 / 2)(N+L+1 / 2)(l+L+\lambda)(l+L+\lambda+1)(l+L-\lambda)(l+L-\lambda-1)}{(2 l-1)(2 l+1)(2 L-1)(2 L+1)}\right]^{1 / 2} \\
& \left\langle n l-1 N L-1 ; \lambda \mid n_{1} l_{1} n_{2} l_{2} ; \lambda\right\rangle_{\beta} .
\end{aligned}
$$

A similar expression with $n_{2}$ incremented by one unit is obtained with the change $n_{1} \rightarrow n_{2}$ in the left hand side of (3.7) and $C \rightarrow-S, S \rightarrow C$ in the right hand side of (3.7). Formulae (3.6) and (3.7) allow us to calculate all the $B M$ coefficients starting with $\langle 0000,0 \mid 0000 ; 0\rangle_{\beta}=1$. From these relations it is also obvious that the $B M$ coefficients are real. 
3.3 SOME PROPERTIES. - Directly from their definition

$$
\left\langle n l N L ; \lambda \mid n_{1} l_{1} n_{2} l_{2} ; \lambda\right\rangle_{\beta}=\int\left[\phi_{n l}^{*}(\mathbf{r}) \phi_{N L}^{*}(\mathbf{R})\right]_{\lambda \mu}\left[\phi_{n_{1} l_{1}}\left(\mathbf{r}_{1}\right) \phi_{n_{2} l_{2}}\left(\mathbf{r}_{2}\right)\right]_{\lambda \mu}\left\{\begin{array}{l}
\mathrm{d} \mathbf{r}_{1} \mathrm{dr}_{2} \\
\mathrm{~d} \mathbf{r} \mathrm{d} \mathbf{R}
\end{array}\right.
$$

the following phase relations can be proved

$$
\begin{aligned}
& \left\langle n l N L ; \lambda \mid n_{2} l_{2} n_{1} l_{1} ; \lambda\right\rangle_{\beta}=(-1)^{l_{1}-\lambda}\left\langle n l N L ; \lambda \mid n_{1} l_{1} n_{2} l_{2} ; \lambda\right\rangle_{\beta+\pi} \\
& \left\langle N L n l ; \lambda \mid n_{1} l_{1} n_{2} l_{2} ; \lambda\right\rangle_{\beta}=(-1)^{L-\lambda}\left\langle n l N L ; \lambda \mid n_{1} l_{1} n_{2} l_{2} ; \lambda\right\rangle_{\beta+\pi} .
\end{aligned}
$$

Applying this twice, and (due to the definition of $\beta$ in (3.4)) taking care of

$$
\left\langle n l N L ; \lambda \mid n_{1} l_{1} n_{2} l_{2} ; \lambda\right\rangle_{\beta+2 \pi}=(-1)^{l+L}\left\langle n l N L ; \lambda \mid n_{1} l_{1} n_{2} l_{2} ; \lambda\right\rangle_{\beta}
$$

one gets

$$
\left\langle N L n l ; \lambda \mid n_{2} l_{2} n_{1} l_{1} ; \lambda\right\rangle_{\beta}=(-1)^{l+l_{1}}\left\langle n l N L ; \lambda \mid n_{1} l_{1} n_{2} l_{2} ; \lambda\right\rangle_{\beta} .
$$

Owing to the fact that the $B M$ are real, by replacing the variables of integration $\mathbf{r}, \mathbf{R}$ by $\mathbf{r}_{1}, \mathbf{r}_{2}$ in (3.8) and then making $\mathbf{r}_{2} \rightarrow-\mathbf{r}_{2}$, the following relations can be obtained

$$
\begin{aligned}
\left\langle n_{1} l_{1} n_{2} l_{2} ; \lambda \mid n l N L ; \lambda\right\rangle_{\beta} & =\left\langle n l N L ; \lambda \mid n_{1} l_{1} n_{2} l_{2} ; \lambda\right\rangle_{-\beta} \\
& =(-1)^{l_{2}+L}\left\langle n l N L ; \lambda \mid n_{1} l_{1} n_{2} l_{2} ; \lambda\right\rangle_{\beta} .
\end{aligned}
$$

Now, when a rotation of angle $\beta$ is followed by another rotation of angle $\gamma$, one obtains a very important composition rule

$$
\left\langle n_{5} l_{5} n_{6} l_{6} ; \lambda \mid n_{1} l_{1} n_{2} l_{2} ; \lambda\right\rangle_{\beta+\gamma}=\sum_{n_{3} l_{3} n_{4} l_{4}}\left\langle n_{5} l_{5} n_{6} l_{6} ; \lambda \mid n_{3} l_{3} n_{4} l_{4} ; \lambda\right\rangle_{\gamma}\left\langle n_{3} l_{3} n_{4} l_{4} ; \lambda \mid n_{1} l_{1} n_{2} l_{2} ; \lambda\right\rangle_{\beta} .
$$

In particular, putting $\gamma=-\beta$ in this equation, the orthogonality relation is recovered

$$
\sum_{n_{3} l_{3} n_{4} l_{4}}\left\langle n_{3} l_{3} n_{4} l_{4} ; \lambda \mid n_{5} l_{5} n_{6} l_{6} ; \lambda\right\rangle_{\beta}\left\langle n_{3} l_{3} n_{4} l_{4} ; \lambda \mid n_{1} l_{1} n_{2} l_{2} ; \lambda\right\rangle_{\beta}=\delta_{n_{1}, n_{5}} \delta_{l_{1}, l_{5}} \delta_{n_{2}, n_{6}} \delta_{l_{2}, l_{6}} .
$$

It is possible to derive also a very important « closure relation ». Let us start with the following integral

$$
I_{l}\left(r, r^{\prime}, \beta\right)=\int \phi_{00}\left(\mathbf{R}_{1}\right) \frac{\delta\left(R_{2}-r^{\prime}\right)}{r^{\prime}} Y_{l m}^{*}\left(\hat{\mathbf{R}}_{2}\right) \phi_{00}\left(\mathbf{r}_{2}\right) \frac{\delta\left(r_{1}-r\right)}{r} Y_{l m}\left(\hat{\mathbf{r}}_{1}\right) \mathrm{d} \mathbf{r}_{1} \mathrm{~d} \mathbf{r}_{2}
$$

where $\mathbf{R}_{1}$ and $\mathbf{R}_{2}$ are the coordinates rotated with an angle $\beta\left(\mathbf{R}_{1}\right.$ and $\mathbf{R}_{2}$ correspond to $\mathbf{r}$ and $\mathbf{R}$ respectively in definition (3.4)).

Noting that

$$
\frac{\delta\left(r_{1}-r\right)}{r} Y_{l m}\left(\hat{\mathbf{r}}_{1}\right)=\sum_{n} U_{n l}(r) \phi_{n l m}\left(\mathbf{r}_{1}\right)
$$

a first expression for calculating $I_{l}$ is readily obtained

$$
I_{l}\left(r, r^{\prime}, \beta\right)=\sum_{n} U_{n l}(r) U_{n l}\left(r^{\prime}\right)\langle 00 n l ; l \mid n l 00 ; l\rangle_{\beta}
$$

Now changing the integration variables $\mathbf{r}_{1}, \mathbf{r}_{2}$ into $\mathbf{R}_{1}$ and $\mathbf{r}_{2}$, some lengthy calculations allow us to write

$$
I_{l}\left(r, r^{\prime}, \beta\right)=4 \pi^{-1 / 2}\left|C^{-3}\right| r r^{\prime} i^{l} j_{l}\left(-i 2 S C^{-2} r r^{\prime}\right) \exp \left[-\frac{1+S^{2}}{2 C^{2}}\left(r^{2}+r^{\prime 2}\right)\right]
$$

(where in (3.8) $C=\cos \frac{\beta}{2} S=\sin \frac{\beta}{2}$ ).

In (3.18) $j_{l}(\rho)$ is the spherical Bessel function of order $l$. Using the derivative definition of $j_{l}$ and the fact that $j_{0}(-i \rho)=\frac{\sinh (\rho)}{\rho}$, and equating (3.17) and (3.18) one gets the following "closure relation " 


$$
\begin{aligned}
\sum_{n} U_{n l}(r) U_{n l}\left(r^{\prime}\right)\langle 00 n l ; l \mid n l 00 ; l\rangle_{\beta}= & \\
& =2 \pi^{-1 / 2} S^{-1}\left|C^{-1}\right| \exp \left[-\frac{1+S^{2}}{2 C^{2}}\left(r^{2}+r^{\prime 2}\right)\right]\left[\rho^{l+1}\left(\frac{1}{\rho} \frac{\mathrm{d}}{\mathrm{d} \rho}\right)^{l} \frac{\sinh \rho}{\rho}\right]_{\rho=2 S C^{-2} r r^{\prime}}
\end{aligned}
$$

a similar expression holds if in the left hand side $\langle 00 n l ; l \mid n l 00 ; l\rangle_{\beta}$ is replaced by $\langle n l 00, l \mid n l 00 ; l\rangle_{\beta}$ and in the right hand side $C \rightarrow S$ and $S \rightarrow-C$. This expression with $l=0$ appears in the exchange normalization kernel of the nucleon-nucleon scattering discussed in the next chapter, and in reference [13] it was used as a convergence test of relation (3.17).

\section{Application to the nucleon-nucleon interaction.}

4.1 The general PROBLEM. - We consider the problem of the scattering of two baryon clusters, each one being formed by three quarks of type $u$ and $d$. In principle each quark is characterized by its colour, isospin, spin and space degree of freedom. However, this paper is concerned with the action of the Pauli principle on intrinsic space coordinates and thus we

$$
\begin{aligned}
& \boldsymbol{R}=\frac{1}{6} \sum_{i=1}^{6} \mathbf{r}_{i} \\
& \left.\begin{array}{l}
\mathbf{x}=\mathbf{r}_{2}-\mathbf{r}_{3} \\
\mathbf{y}=3^{-1 / 2}\left(\mathbf{r}_{2}+\mathbf{r}_{3}-2 \mathbf{r}_{1}\right)
\end{array}\right\} \\
& \mathbf{x}^{\prime}=\mathbf{r}_{5}-\mathbf{r}_{6} \\
& \left.y^{\prime}=3^{-1 / 2}\left(r_{5}+r_{6}-2 r_{4}\right)\right\} \\
& \mathbf{R}=3^{-1 / 2}\left(r_{1}+r_{2}+r_{3}-r_{4}-r_{5}-r_{6}\right) \quad \text { separation distance of the clusters . }
\end{aligned}
$$

Taking fully into account the Pauli principle, the trial wave function reads

$$
\Psi\left(\mathbf{r}_{1}, \mathbf{r}_{2}, \mathbf{r}_{3}, \mathbf{r}_{4}, \mathbf{r}_{5}, \mathbf{r}_{6}\right)=\psi\left(\mathbf{x}, \mathbf{y}, \mathbf{x}^{\prime}, \mathbf{y}^{\prime}, \mathbf{R}, \mathfrak{R}\right)=\mathcal{A} f_{\text {C.M. }}(\mathfrak{R}) \varphi_{\mathrm{N}}(\mathbf{x}, \mathbf{y}) \varphi_{\mathrm{N}}\left(\mathbf{x}^{\prime}, \mathbf{y}^{\prime}\right) \chi(\mathbf{R})
$$

where $f_{\text {C.M. }}, \phi_{N}, \chi$ are the centre of mass, intrinsic cluster, relative wave function, respectively and $\mathcal{A}$ the total antisymmetrizer. The centre of mass function, being symmetric in the 6-q coordinates, can be kept out of the antisymmetrizer and is easily dealt with. It will be ignored in the following. Let $T$ be any operator symmetric in the exchange of the particles. The main problem is the calculation of quantity $\langle\psi|T| \psi\rangle$. Since $\mathcal{A}^{+}=\mathcal{A}$, $\mathcal{A}^{2}=\mathcal{A}$ and $\mathcal{A T A}=T$ it is well known that it is sufficient to keep the antisymmetrizer on the bra vector only. Moreover, the intrinsic functions $\phi_{N}$ are built antisymmetric for all permutations of the subgroup $S_{3} \times S_{3} \subset S_{6}$. This property allows the number of different contributions to the average $\langle\psi|T| \psi\rangle$ to be reduced from 720 to 2 ; one can choose for instance 1 (identity) and $P_{14}$ (transposition of particles 1 and 4) and the corresponding contributions are denoted as direct and exchange contributions. In general the direct part is easy to calculate and the main problem in cluster theory lies in the evaluation of the exchange parts. If it is possible to expand the trial intrinsic wave function in terms of the harmonic oscillator basis $\phi(r)$, this problem reduces to the computation of

$$
\left\langle\varphi\left(\mathbf{x}_{\mathbf{P}}\right) \varphi\left(\mathbf{y}_{\mathbf{P}}\right) \varphi\left(\mathbf{x}_{\mathbf{P}}^{\prime}\right) \varphi\left(\mathbf{y}_{\mathbf{P}}^{\prime}\right) \varphi\left(\mathbf{R}_{\mathbf{P}}\right)|T| \varphi(\mathbf{x}) \varphi(\mathbf{y}) \varphi\left(\mathbf{x}^{\prime}\right) \varphi\left(\mathbf{y}^{\prime}\right) \varphi(\mathbf{R})\right\rangle .
$$

In this expression the permuted coordinates $\mathbf{x}_{\mathrm{p}}$, $\mathbf{y}_{\mathrm{P}}, \mathbf{x}_{\mathrm{P}}^{\prime}, \mathbf{y}_{\mathrm{P}}^{\prime}, \mathbf{R}_{\mathrm{P}}$ are those obtained in definition (4.1) by permuting $\mathbf{r}_{1} \leftrightarrow \mathbf{r}_{\mathbf{4}}$. From the conclusions of sections 2 and 3 , one sees that this quantity is obtained with a summation of a finite number of terms including generalized Brody-Moshinsky coefficients. In the following we apply these general ideas for $T=1$ (exchange normalization contribution) and for $T=V_{\text {int }}$ (exchange potential contribution). In fact, among the 9 terms coming from the cluster interaction, only three give different contributions and one can write formally

$$
V_{\text {int }}=V_{14}+4 V_{15}+4 V_{25} .
$$

\subsection{EXCHANGE NORMALIZATION CONTRIBUTION. -} Since

$$
\begin{aligned}
& \mathbf{x}_{\mathbf{P}}=\mathbf{r}_{2}-\mathbf{r}_{3}=\mathbf{x} \\
& \mathbf{x}_{\mathbf{P}}^{\prime}=\mathbf{r}_{5}-\mathbf{r}_{6}=\mathbf{x}^{\prime}
\end{aligned}
$$




$$
\begin{aligned}
& \mathbf{y}_{\mathbf{P}}=3^{-1 / 2}\left(\mathbf{r}_{2}+\mathbf{r}_{3}-2 \mathbf{r}_{4}\right)=3^{-1}\left[2 \mathbf{R}+\mathbf{y}+2 \mathbf{y}^{\prime}\right] \\
& \mathbf{y}_{\mathbf{P}}^{\prime}=3^{-1 / 2}\left(\mathbf{r}_{5}+\mathbf{r}_{6}-2 \mathbf{r}_{1}\right)= 3^{-1}\left[-2 \mathbf{R}+2 \mathbf{y}+\mathbf{y}^{\prime}\right] \\
& \mathbf{R}_{\mathbf{P}}=3^{-1 / 2}\left(\mathbf{r}_{4}+\mathbf{r}_{2}+\mathbf{r}_{3}-\mathbf{r}_{1}-\mathbf{r}_{5}-\mathbf{r}_{6}\right) \\
&=3^{-1}\left[\mathbf{R}+2 \mathbf{y}-2 \mathbf{y}^{\prime}\right]
\end{aligned}
$$

it is trivial that quantity (4.3) with $T=\mathbb{1}$ (called $\mathcal{N}$ ) immediately reduces to (the integration on $\mathbf{x}$ and $\mathbf{x}^{\prime}$ variables gives $\delta$ symbols which are omitted in the following)

$$
\mathcal{N} \rightarrow\left\langle\varphi\left(\mathbf{y}_{\mathbf{P}}\right) \varphi\left(\mathbf{y}_{\mathbf{P}}^{\prime}\right) \varphi\left(\mathbf{R}_{\mathbf{P}}\right) \mid \varphi(\mathbf{y}) \varphi\left(\mathbf{y}^{\prime}\right) \varphi(\mathbf{R})\right\rangle .
$$

Keeping in a first step the coordinates $\mathbf{R}$ and $\mathbf{R}_{\mathrm{P}}$ fixed and making a "standard " rotation in the $\left(\mathbf{y}, \mathbf{y}^{\prime}\right)$ plane and in the $\left(\mathbf{y}_{\mathrm{P}}, \mathbf{y}_{\mathrm{P}}^{\prime}\right)$ plane one introduces the new coordinates

$$
\begin{aligned}
& \mathbf{u}=2^{-1 / 2}\left(\mathbf{y}+\mathbf{y}^{\prime}\right)=2^{-1 / 2}\left(\mathbf{y}_{\mathbf{P}}+\mathbf{y}_{\mathbf{P}}^{\prime}\right)=\mathbf{u}_{\mathbf{P}} \\
& \mathbf{v}=2^{-1 / 2}\left(\mathbf{y}-\mathbf{y}^{\prime}\right) \\
& \mathbf{v}_{\mathbf{P}}=2^{-1 / 2}\left(\mathbf{y}_{\mathbf{P}}-\mathbf{y}_{\mathbf{P}}^{\prime}\right) .
\end{aligned}
$$

This allows, through the introduction of two standard $B M$, expression (4.6) to be changed into

$$
\mathcal{N} \rightarrow\left\langle\varphi(\mathbf{u}) \varphi\left(\mathbf{v}_{\mathbf{P}}\right) \varphi\left(\mathbf{R}_{\mathbf{P}}\right) \mid \varphi(\mathbf{u}) \varphi(\mathbf{v}) \varphi(\mathbf{R})\right\rangle
$$

or, after trivial integration on the $\mathbf{u}$ variable, into

$$
\mathcal{N} \rightarrow\left\langle\varphi\left(\mathbf{v}_{\mathbf{p}}\right) \varphi\left(\mathbf{R}_{\mathbf{P}}\right) \mid \varphi(\mathbf{v}) \varphi(\mathbf{R})\right\rangle .
$$

It is easy to show that

$$
\mathbf{R}_{\mathbf{P}}=\frac{\sqrt{8}}{3} \mathbf{v}+\frac{1}{3} \mathbf{R} \text { and } \mathbf{v}_{\mathbf{P}}=-\frac{1}{3} \mathbf{v}+\frac{\sqrt{8}}{3} \mathbf{R}
$$

then $\mathbf{R}_{\mathrm{P}}$ and $\mathbf{v}_{\mathrm{P}}$ are derived from $\mathbf{v}$ and $\mathbf{R}$ by a rotation of angle $\beta_{1}$ such that $C=\sqrt{8} / 3$ and $S=1 / 3$; consequently (4.8) is nothing else that a generalized $B M$ with angle $\beta_{1}$. Thus expression (4.6) can be written very symbolically

$$
\mathcal{N}=\sum B M \cdot B M \cdot B M_{\beta_{1}}
$$

meaning that it is a summation of a finite number of terms including 3 different Brody-Moshinsky coefficients (two standard coefficients and one with angle $\beta_{1}$ ). We think that this is the most economical procedure for calculating (4.6).

4.3 Exchange $V_{14}$ CONTRIBUtion. - The problem here is the evaluation of

$$
\vartheta_{14}=\left\langle\varphi\left(\mathbf{x}_{\mathbf{P}}\right) \varphi\left(\mathbf{y}_{\mathbf{P}}\right) \varphi\left(\mathbf{x}_{\mathbf{P}}^{\prime}\right) \varphi\left(\mathbf{y}_{\mathbf{P}}^{\prime}\right) \varphi\left(\mathbf{R}_{\mathbf{P}}\right)\left|V_{14}\left(\mathbf{r}_{1}-\mathbf{r}_{4}\right)\right| \varphi(\mathbf{x}) \varphi(\mathbf{y}) \varphi\left(\mathbf{x}^{\prime}\right) \varphi\left(\mathbf{y}^{\prime}\right) \varphi(\mathbf{R})\right\rangle .
$$

Due to the fact that $V_{14}$ depends on the coordinate $\mathbf{r}=\mathbf{r}_{1}-\mathbf{r}_{4}$ only, it is natural to impose that this variable must be one of the final integration variables. For instance one can choose the following coordinates

$$
\begin{gathered}
\mathbf{x} ; \mathbf{x}^{\prime} ; \mathbf{r}=\mathbf{r}_{1}-\mathbf{r}_{4} \\
\mathbf{z}=2^{-1 / 2}\left(\mathbf{r}_{2}+\mathbf{r}_{3}-\mathbf{r}_{5}-\mathbf{r}_{6}\right) \\
\mathbf{u}=6^{-1 / 2}\left(\mathbf{r}_{2}+\mathbf{r}_{3}+\mathbf{r}_{5}+\mathbf{r}_{6}-2 \mathbf{r}_{1}-2 \mathbf{r}_{4}\right)
\end{gathered}
$$

Since $\mathbf{x}_{\mathrm{P}}=\mathbf{x}$ and $\mathbf{x}_{\mathrm{P}}^{\prime}=\mathbf{x}^{\prime}$, the integration over the $\mathbf{x}$ and $x^{\prime}$ variables reduces $v_{14}$ to

$$
\vartheta_{14} \rightarrow\left\langle\varphi\left(\mathbf{y}_{\mathbf{P}}\right) \varphi\left(\mathbf{y}_{\mathbf{P}}^{\prime}\right) \varphi\left(\mathbf{R}_{\mathbf{P}}\right)|V(\mathbf{r})| \varphi(\mathbf{x}) \varphi(\mathbf{y}) \varphi(\mathbf{R})\right\rangle .
$$

Keeping the $\mathbf{R}$ and $\mathbf{R}_{\mathrm{P}}$ coordinates fixed and making $a$ « standard " rotation on the $\left(\mathbf{y}, \mathbf{y}^{\prime}\right)$ plane and $\left(\mathbf{y}_{\mathrm{p}}, \mathbf{y}_{\mathrm{p}}^{\prime}\right)$ plane, one can define the new variables

$$
\begin{aligned}
& \mathbf{u}=2^{-1 / 2}\left(\mathbf{y}+\mathbf{y}^{\prime}\right)=2^{-1 / 2}\left(\mathbf{y}_{\mathbf{P}}+\mathbf{y}_{\mathbf{p}}^{\prime}\right)=\mathbf{u}_{\mathrm{p}} \\
& \mathbf{v}=2^{-1 / 2}\left(\mathbf{y}-\mathbf{y}^{\prime}\right)=3^{-1}(-\sqrt{6} \mathbf{r}+\sqrt{3} \mathbf{z}) \\
& \mathbf{v}_{\mathbf{P}}=2^{-1 / 2}\left(\mathbf{y}_{\mathbf{P}}-\mathbf{y}_{\mathbf{P}}^{\prime}\right)=3^{-1}(\sqrt{6} \mathbf{r}+\sqrt{3} \mathbf{z}) .
\end{aligned}
$$

Hence with the help of two standard $B M$ and inte- gration on $\mathbf{u}$ variable, $\vartheta_{14}$ now reduces to

$$
\vartheta_{14} \rightarrow\left\langle\varphi\left(\mathbf{v}_{\mathbf{p}}\right) \varphi\left(\mathbf{R}_{\mathbf{P}}\right)|V(\mathbf{r})| \varphi(\mathbf{v}) \varphi(\mathbf{R})\right\rangle .
$$

Since, on the other hand,

and

$$
\mathbf{R}=3^{-1}(\sqrt{3} \mathbf{r}+\sqrt{6} \mathbf{z})
$$

$$
\mathbf{R}_{\mathbf{P}}=3^{-1}(-\sqrt{3} \mathbf{r}+\sqrt{6} \mathbf{z}),
$$

it is evident that $(\mathbf{R}, \mathbf{v})$ is deduced from $(\mathbf{r}, \mathbf{z})$ by a rotation through an angle $\beta_{2}$ such that $C=\sqrt{1 / 3}$, $S=\sqrt{2 / 3}$; similarly $\mathbf{v}_{\mathrm{P}}$ and $-\mathbf{R}_{\mathrm{P}}$ are obtained from $\mathbf{z}$ and $\mathbf{r}$ through the same rotation. Then, using two $B M_{\beta_{2}}$, the scalar product (4.15) is transformed into

$$
\vartheta_{14} \rightarrow\langle\varphi(\mathbf{r}) \varphi(\mathbf{z})|V(\mathbf{r})| \varphi(\mathbf{r}) \varphi(\mathbf{z})\rangle
$$

which is almost trivial. All this can be summarized by

$$
\bigcup_{14}=\sum B M \cdot B M \cdot B M_{\beta_{2}} \cdot B M_{\beta_{2}} \cdot
$$

In this case again we think that this procedure is the «best one ". 
4.4 EXCHANGE $V_{15}$ CONTRIBUtion. - This requires the evaluation of

$$
\vartheta_{15}=\left\langle\varphi\left(\mathbf{x}_{\mathbf{P}}\right) \varphi\left(\mathbf{y}_{\mathbf{P}}\right) \varphi\left(\mathbf{x}_{\mathbf{P}}^{\prime}\right) \varphi\left(\mathbf{y}_{\mathbf{P}}^{\prime}\right) \varphi\left(\mathbf{R}_{\mathbf{P}}\right)\left|V_{15}\left(\mathbf{r}_{1}-\mathbf{r}_{5}\right)\right| \varphi(\mathbf{x}) \varphi(\mathbf{y}) \varphi\left(\mathbf{x}^{\prime}\right) \varphi\left(\mathbf{y}^{\prime}\right) \varphi(\mathbf{R})\right\rangle .
$$

As already stated, one must keep $\mathbf{r}=\mathbf{r}_{1}-\mathbf{r}_{5}$ as an integration variable; it is easy to show that $\mathbf{x}$ can be chosen as well. In a first choice we propose to take

$$
\mathbf{x} ; \mathbf{r}=\mathbf{r}_{1}-\mathbf{r}_{5} ; \mathbf{y}_{\mathrm{P}}, \mathbf{R}_{\mathrm{P}} \quad \text { and } \quad \mathbf{z}=3^{-1 / 2}\left(\mathbf{r}_{1}+\mathbf{r}_{5}-2 \mathbf{r}_{6}\right)
$$

as integration variables. Since $\mathbf{x}_{\mathbf{P}}=\mathbf{x}$, we are left with

$$
\mho_{15} \rightarrow\left\langle\varphi\left(\mathbf{y}_{\mathbf{P}}\right) \varphi\left(\mathbf{R}_{\mathbf{P}}\right) \varphi\left(\mathbf{x}^{\prime}\right) \varphi\left(\mathbf{y}_{\mathbf{P}}^{\prime}\right)|V(\mathbf{r})| \varphi\left(\mathbf{x}^{\prime}\right) \varphi(\mathbf{y}) \varphi\left(\mathbf{y}^{\prime}\right) \varphi(\mathbf{R})\right\rangle .
$$

One verifies easily that

$$
\begin{aligned}
& \mathbf{x}^{\prime}=-\frac{\mathbf{r}}{2}+\frac{\sqrt{3}}{2} \mathbf{z} \\
& \mathbf{y}_{\mathbf{P}}^{\prime}=-\frac{\sqrt{3}}{2} \mathbf{r}-\frac{\mathbf{z}}{2} .
\end{aligned}
$$

Introducing new variables as

$$
\begin{aligned}
& \mathbf{u}=\frac{1}{\sqrt{5}} \mathbf{y}-\frac{2}{\sqrt{5}} \mathbf{y}^{\prime}=\frac{2}{\sqrt{5}} \mathbf{R}_{\mathbf{P}}-\frac{1}{\sqrt{5}} \mathbf{y}_{\mathrm{P}} \\
& \mathbf{v}=\frac{2}{\sqrt{5}} \mathbf{y}+\frac{1}{\sqrt{5}} \mathbf{y}^{\prime}=\frac{\sqrt{5}}{30}\left(4 \mathbf{R}_{\mathbf{P}}-5 \sqrt{3} \mathbf{r}+8 \mathbf{y}_{\mathbf{P}}-5 \mathbf{z}\right)
\end{aligned}
$$

and, with the angle $\beta_{4}$ such that $C=\frac{2}{3}, S=\frac{\sqrt{5}}{3}$, with the help of $B M_{\beta_{4}}, \phi(\mathbf{v}) \phi(\mathbf{R})$ is transformed into $\phi(\mathbf{s}) \phi(\mathbf{t})$.

Now let us put

$$
\begin{aligned}
& \mathbf{s}=\frac{1}{\sqrt{5}} \mathbf{R}_{\mathrm{P}}+\frac{2}{\sqrt{5}} \mathbf{y}_{\mathrm{P}} \\
& \mathbf{t}=-\mathbf{y}_{\mathrm{P}}^{\prime}=\frac{\sqrt{3}}{2} \mathbf{r}+\frac{1}{2} \mathbf{z}
\end{aligned}
$$

it is trivial to show that

$$
\begin{aligned}
& \mathbf{v}=\frac{2}{3} s-\frac{\sqrt{5}}{3} t \\
& R=\frac{\sqrt{5}}{3} s+\frac{2}{3} t
\end{aligned}
$$

and, with the angle $\beta_{4}$ such that $C=\frac{2}{3}, S=\frac{\sqrt{5}}{3}$, with the help of $B M_{\beta 4}, \phi(\mathbf{v}) \phi(\mathbf{R})$ is transformed into $\phi(\mathbf{s}) \phi(\mathbf{t})$. At this stage we are left with the evaluation of

$$
\vartheta_{15} \rightarrow\left\langle\varphi\left(\mathbf{y}_{\mathbf{P}}\right) \varphi\left(\mathbf{R}_{\mathbf{P}}\right) \varphi\left(\mathbf{x}^{\prime}\right) \varphi(\mathbf{t})|V(\mathbf{r})| \varphi(\mathbf{u}) \varphi(\mathbf{s}) \varphi\left(\mathbf{x}^{\prime}\right) \varphi(\mathbf{t})\right\rangle .
$$

Lastly from (4.23) and (4.24) one sees that a rotation with angle $\beta_{3}$ transforms $\phi(\mathbf{u}) \phi(\mathbf{s})$ into $\phi\left(\mathbf{y}_{\mathrm{p}}\right) \phi\left(\mathbf{R}_{\mathrm{p}}\right)$ and the rotation with angle $\beta_{5}$ such that $C=\frac{\sqrt{3}}{2}, S=\frac{1}{2}$ transforms $\phi(\mathbf{t}) \phi\left(\mathbf{x}^{\prime}\right)$ into $\phi(\mathbf{r}) \phi(\mathbf{z})$ which achieves the reduction. Symbolically one has

$$
\bigcup_{15}=\sum B M_{\beta 3} \cdot B M_{\beta 4} \cdot B M_{\beta_{3}} \cdot B M_{\beta 5} \cdot B M_{\beta 5} .
$$

In this peculiar case, we think that this procedure is not necessarily the best; let us illustrate that there is an 
alternative procedure which more or less exhibits the same levels of difficulty. We choose, as integration variables

$$
\begin{gathered}
x ; \quad r=r_{1}-r_{5} ; \quad s=3^{-1 / 2}\left(r_{1}+r_{5}-2 r_{6}\right) \\
z=6^{-1 / 2}\left(r_{1}+r_{5}+r_{6}-3 r_{4}\right) ; \quad v=6^{-1 / 2}\left(r_{1}+r_{4}+r_{5}+r_{6}-2 r_{2}-2 r_{3}\right) .
\end{gathered}
$$

It follows that a « standard rotation » transforms $(\mathbf{y}, \mathbf{R})$ into $(-\mathbf{v}, \mathbf{u})$ with

$$
\mathbf{u}=\frac{1}{3} \mathbf{z}-\frac{\sqrt{2}}{3} \mathbf{s}-\frac{\sqrt{6}}{3} \mathbf{r}
$$

the same rotation transforms $\phi\left(\mathbf{y}_{\mathbf{p}}\right) \phi\left(\mathbf{R}_{\mathbf{p}}\right)$ into $\phi(\mathbf{z}) \phi(-\mathbf{v})$. Moreover, a rotation with angle $\beta_{1}$ as defined by (4.9) allows us to go from $\phi(-\mathbf{u}) \phi\left(\mathbf{y}^{\prime}\right)$ to $\phi(\mathbf{w}) \phi(\mathbf{z})$ where

$$
\mathbf{w}=\frac{\mathbf{s}}{2}+\frac{\sqrt{3}}{2} \mathbf{r}=-\mathbf{y}_{\mathbf{P}}^{\prime}
$$

Lastly a rotation with angle $\beta_{5}=\pi / 3$ transforms $\phi(\mathbf{w}) \phi\left(\mathbf{x}^{\prime}\right)$ into $\phi(\mathbf{r}) \phi(\mathbf{s})$. The same rotation also transforms $\phi\left(-\mathbf{y}_{\mathbf{p}}^{\prime}\right) \phi\left(\mathbf{x}^{\prime}\right)$ into $\phi(\mathbf{r}) \phi(\mathbf{s})$. Consequently the calculation of $\mho_{15}$ can be summarized symbolically by

$$
\vartheta_{15}=\sum B M \cdot B M_{\beta_{1}} \cdot B M \cdot B M_{\beta 5} \cdot B M_{\beta 5} .
$$

If we compare (4.31) to (4.27), it appears that both computations are of equal difficulty. This example clearly exhibits that the decomposition of the original quantity into successive planar rotations may be obtained by several equivalent ways. The procedure to be chosen depends on each particular case considered.

4.5 EXChANGE $V_{25}$ CONTRIBUtion. - In that case one must calculate

$$
\vartheta_{25}=\left\langle\varphi\left(\mathbf{x}_{\mathbf{P}}\right) \varphi\left(\mathbf{y}_{\mathbf{P}}\right) \varphi\left(\mathbf{x}_{\mathbf{P}}^{\prime}\right) \varphi\left(\mathbf{y}_{\mathbf{P}}^{\prime}\right) \varphi\left(\mathbf{R}_{\mathbf{P}}\right)\left|V_{25}\left(\mathbf{r}_{2}-\mathbf{r}_{5}\right)\right| \varphi(\mathbf{x}) \varphi(\mathbf{y}) \varphi\left(\mathbf{x}^{\prime}\right) \varphi\left(\mathbf{y}^{\prime}\right) \varphi(\mathbf{R})\right\rangle .
$$

As already stated, it is necessary to keep $\mathbf{r}_{2}-\mathbf{r}_{5}=\mathbf{r}$ as an integration variable. In this case, which is the most complicated (because one cannot keep $\mathbf{x}$ as integration variable), we found convenient to define the following integration variables

$$
\begin{gathered}
\mathbf{r}=\mathbf{r}_{2}-\mathbf{r}_{5} ; \quad \mathbf{r}_{36}=\mathbf{r}_{3}-\mathbf{r}_{6} ; \quad r_{14}=r_{1}-\mathbf{r}_{4} \\
\mathbf{z}=2^{-1 / 2}\left(\mathbf{r}_{2}+\mathbf{r}_{5}-\mathbf{r}_{3}-\mathbf{r}_{6}\right) ; \quad \mathbf{u}=6^{-1 / 2}\left(\mathbf{r}_{2}+\mathbf{r}_{3}+\mathbf{r}_{5}+\mathbf{r}_{6}-2 \mathbf{r}_{1}-2 \mathbf{r}_{4}\right) .
\end{gathered}
$$

They are related to the original ones by

$$
\begin{aligned}
& \mathbf{x}=+\frac{1}{2}\left(\mathbf{r}-\mathbf{r}_{36}\right)+\frac{\sqrt{2}}{2} \mathbf{z} \\
& \mathbf{x}^{\prime}=-\frac{\sqrt{3}}{6}\left(\mathbf{r}+\mathbf{r}_{36}-2 \mathbf{r}_{14}\right)+\frac{\sqrt{2}}{2} \mathbf{u} \\
& \mathbf{y}=+\frac{\sqrt{2}}{\mathbf{y}^{\prime}}=-\frac{\sqrt{3}}{2} \mathbf{u} \\
& \mathbf{y}_{\mathbf{P}}= \pm \frac{\sqrt{3}}{6}\left(\mathbf{r}+\mathbf{r}_{36}+2 \mathbf{r}_{14}\right)+\frac{\sqrt{3}}{\mathbf{y}_{\mathbf{P}}^{\prime}}=- \\
& \mathbf{R}=+\frac{\sqrt{3}}{3} \mathbf{r}_{14}+\frac{\sqrt{3}}{3}\left(\mathbf{r}+\mathbf{r}_{36}\right) . \\
& \mathbf{R}_{\mathbf{P}}=-
\end{aligned}
$$

Noting that $\mathbf{z}=\frac{1}{\sqrt{2}}\left(\mathbf{x}+\mathbf{x}^{\prime}\right)$ and defining

$$
\mathbf{w}=2^{-1 / 2}\left(\mathbf{x}-\mathbf{x}^{\prime}\right)=2^{-1 / 2}\left(\mathbf{r}-\mathbf{r}_{36}\right)
$$

one transforms $\phi(\mathbf{x}) \phi\left(\mathbf{x}^{\prime}\right)$ (and $\left.\phi\left(\mathbf{x}_{\mathrm{p}}\right) \phi\left(\mathbf{x}_{\mathrm{p}}^{\prime}\right)\right)$ into $\phi(\mathbf{z}) \phi(\mathbf{w})$ with the help of a standard $B M$. Now in the same manner

$$
\begin{aligned}
& \mathbf{u}=2^{-1 / 2}\left(\mathbf{y}+\mathbf{y}^{\prime}\right)=\mathbf{u}_{\mathbf{p}} \\
& \mathbf{v}=2^{-1 / 2}\left(\mathbf{y}-\mathbf{y}^{\prime}\right)=6^{-1 / 2}\left(\mathbf{r}+\mathbf{r}_{36}-2 \mathbf{r}_{14}\right) \\
& \mathbf{v}_{\mathbf{p}}=2^{-1 / 2}\left(\mathbf{y}_{\mathbf{P}}-\mathbf{y}_{\mathbf{P}}^{\prime}\right)=6^{-1 / 2}\left(\mathbf{r}+\mathbf{r}_{36}+2 \mathbf{r}_{14}\right)
\end{aligned}
$$


we obtain $\phi(\mathbf{u}) \phi(\mathbf{v})\left(\right.$ and $\left.\phi(\mathbf{u}) \phi\left(\mathbf{v}_{\mathbf{p}}\right)\right)$ from $\phi(\mathbf{y}) \phi\left(\mathbf{y}^{\prime}\right)$ (and $\left.\phi\left(\mathbf{y}_{\mathbf{P}}\right) \phi\left(\mathbf{y}_{\mathbf{P}}^{\prime}\right)\right)$ by a standard $B M$. After integration on the $\mathbf{u}$ and $\mathbf{z}$ variables we are left with

$$
\vartheta_{25} \rightarrow\left\langle\varphi(\mathbf{w}) \varphi\left(\mathbf{v}_{\mathbf{P}}\right) \varphi\left(\mathbf{R}_{\mathbf{P}}\right)|V(\mathbf{r})| \varphi(\mathbf{w}) \varphi(\mathbf{v}) \varphi(\mathbf{R})\right\rangle .
$$

It is not difficult to see that a rotation with angle $\beta_{2}$ transforms $\mathbf{v}$ and $\mathbf{R}$ (and $\mathbf{v}_{\mathbf{P}}$ and $\mathbf{R}_{\mathrm{P}}$ ) into $\mathbf{s}=\frac{1}{\sqrt{2}}\left(\mathbf{r}+\mathbf{r}_{36}\right.$ ) and $\mathbf{r}_{14}$ (s and $\left.-\mathbf{r}_{14}\right)$; hence with a $B M_{\beta_{2}}$ we go from $\phi(\mathbf{v}) \phi(\mathbf{R})\left(\right.$ and $\left.\phi\left(\mathbf{v}_{\mathbf{p}}\right) \phi\left(\mathbf{R}_{\mathrm{P}}\right)\right)$ to $\phi(\mathbf{s}) \phi\left(\mathbf{r}_{14}\right)$. Finally a standard $B M$ transforms $\phi(\mathbf{w}) \phi(\mathbf{s})$ into $\phi(\mathbf{r}) \phi\left(\mathbf{r}_{36}\right)$. Symbolically this $V_{25}$ contribution is written

$$
\vartheta_{25}=\sum B M \cdot B M \cdot B M . B M \cdot B M_{\beta_{2}} \cdot B M_{\beta_{2}} \cdot B M \cdot B M .
$$

The various decompositions presented here were the underlying technical ingredients of reference [13]. They allow the introduction of radial excitations of the nucleon in the description of nucleon-nucleon scattering. More complete formulae including spin-isospin components can be found in the internal report [14].

\section{Conclusions.}

In this paper, we studied the properties of the generalized Brody-Moshinsky coefficients in detail; in the literature up to now, only the standard coefficients have deserved some work but here, all the coefficients are treated on equal footing. A new closure relation, which appeared to be important in the microscopic description of the nucleon-nucleon interaction, was derived. On the other hand, it is shown that every set of permuted intrinsic coordinates is related to another one by an orthogonal transformation. This property is very important for the cluster models which rigorously eliminate the centre of mass motion. If it is possible to expand the kernels coming from the Schrödinger equation (in most cases, this equation is of the Hill-Wheeler type) in terms of a harmonic oscillator basis with sufficiently rapid convergence, then our work shows that it is possible to calculate exactly the kernels - specially the complicated exchange kernels - with a finite number of terms which include generalized Brody-Moshinsky coefficients. The practi- cal way - that is the most economical one - to do that depends on each particular system which is considered. This method should be convenient for the study of light systems which could not be solved exactly up to now (let say with a particule number between 4 and 8). The application to the nucleonnucleon interaction described in terms of the constituent quarks (6 particles) has been abundantly discussed. This method opens new possibilities in the cluster theory; in particular it is possible to retain the radial excitations of the nucleon in the description of the nucleon-nucleon scattering.

\section{Acknowledgments.}

We are very much indebted to Prof. C. Gignoux for constant interest in this work and numerous illuminating discussions. We are also grateful to Dr. A. K. Jain for suggesting the second possibility of evaluating $V_{15}$, and to Drs. J. Carbonell and S. Shlomo for a careful reading of the manuscript.
[1] Peierls, R. E. and Yoccoz, J. Proc. Phys. Soc. A 70 (1957) 381.

Peierls, R. E. and Thouless, D., J. Nucl. Phys. 38 (1962) 154.

[2] Wildermuth, K. and TANG, Y. C., A unified theory of the nucleus (Vieweg) 1977.

WildeRmuth, K. and MCCluRE, W., Cluster representations of nuclei (Springer-Verlag) 1966.

Kukulin, V. I., Neudatchin, V. G., OBukhovski, I. T. and SmIRnov, Y. F., Cluster as subsystems in light nuclei (Vieweg) 1983.

[3] Bencze, Gy. and Redish, E. F., Nucl. Phys. A 238 (1975) 240.

Bencze, Gy and Chandler, C., Phys. Rev. C 25 (1982) 136.
[4] Merkuriev, S., Yakovlev, S. L. and Gignoux, C., Nucl. Phys. A 431 (1984) 125.

[5] Moshinsky, M., Nucl. Phys. 13 (1959) 104.

[6] Erdelyi, A., Magnus, W., Oberhettinger, F. and Tricomi, F. G., Higher transcendental functions (Mc Graw-Hill) Vol. 2, 1953, p. 188.

[7] Brody, T. A. and Moshinksy, M., Tables of transformation brackets for nuclear shell model calculations (2nd edit.) (Gordon \& Breach), 1967.

Numerous references concerning standard BrodyMoshinsky coefficients can be found in LAwson, R. D., Theory of the nuclear shell model (Oxford University Press) 1980, Appendix 6.

[8] Moshinsky, M., Cargese lectures in Physics, ed. M. Jean (1969) Vol. 3. 
[9] Arima, A. and Teresawa, T., Prog. Theor. Phys. 23 (1960) 115.

[10] Smirnov, Y. F., Nucl. Phys. 27 (1961) 177.

Bakri, M. M., Nucl. Phys. A 96 (1967) 115.

[11] Talman, J. D. and Lande, A., Nucl. Phys. A 163 (1971) 249.
[12] Raynal, J., Nucl. Phys. A 259 (1976) 272.

[13] Silvestre-Brac, B., Jain, A. K. and Gignoux, C., Phys. Lett. 137B (1984) 5.

[14] Silvestre-Brac, B., Jain, A. K. and Gignoux, C., Internal Report ISN 83-56.

[15] Talmi, I., Helv. Phys. Acta 25 (1952) 185. 